\title{
BMJ Open Mindfulness-based and cognitive-based stress prevention in student teachers (startklar): study protocol of a randomised controlled trial
}

\author{
Jasmina Eskic, Sophie Merle Kuhlmann, Katharina Kreinbihl, Florian Hammerle
}

To cite: Eskic J, Kuhlmann SM, Kreinbihl K, et al. Mindfulnessbased and cognitive-based stress prevention in student teachers (startklar): study protocol of a randomised controlled trial. BMJ Open 2019;9:e021941. doi:10.1136/ bmjopen-2018-021941

- Prepublication history for this paper is available online. To view these files please visit the journal online (http://dx.doi org/10.1136/bmjopen-2018021941).

Received 30 January 2018 Revised 18 0ctober 2018 Accepted 2 November 2018

Check for updates

(c) Author(s) (or their employer(s)) 2019. Re-use permitted under CC BY-NC. No commercial re-use. See rights and permissions. Published by BMJ.

Department of Child and Adolescent Psychiatry and Psychotherapy, University Medical Center of the Johannes Gutenberg University Mainz, Mainz, Germany

\section{Correspondence to} Jasmina Eskic; jasmina.eskic@unimedizinmainz.de

\section{ABSTRACT}

Introduction Teachers and student teachers in Germany are a high-risk population for stress and stress-related mental health problems. This often leads to early retirement in subsequent professional life. Various trials have demonstrated positive effects of stress prevention training on the perceived stress and stress-related symptoms of teachers. Although many studies show positive effects of mindfulness-based stress interventions, there is not yet any mindfulness-based intervention for teachers or student teachers in Germany. The aim of this trial is to evaluate a training that combines mindfulnessbased and cognitive interventions into one programme, addressing to the specific burdens of student teachers. Methods and analysis This study protocol presents a prospective block-randomised controlled trial. Assessment will take place at three time points (baseline, postintervention, 3-month follow-up) for an intervention and waiting control group, and at a fourth assessment point for the waiting control group after receiving the training. The aim is to evaluate the effects of mindfulness-based stress prevention on stress, psychological morbidity, burnout and self-efficacy using validated measures. Participants are student teachers from German teacher training colleges and participation will be voluntary. The targeted total sample size is 96 at 3-month follow-up. The training will comprise three 4-hour sessions conducted every 2 weeks. The control group will participate in the training after the 3-month follow-up. The allotment will be randomised with a stratified allocation ratio by gender. After descriptive statistics have been evaluated, inferential statistical analysis will be conducted using repeated measures analysis of variance with interactions between time and group. Effect sizes will be calculated using partial $\eta^{2}$ values

Ethics and dissemination Results will be disseminated at conferences, in specialist magazines and through peerreviewed publications. The trial has been approved by the ethics review board of the local medical association, Mainz, Germany, under the reference number 837.192.16 (10511).

Trial registration number DRKS00010897.

\section{INTRODUCTION}

Research shows that teachers and student teachers in Germany are a high-risk population
Strengths and limitations of this study

- First training in Germany to combine mindfulness-based and cognitive-behavioural-based strategies for stress prevention in student teachers.

- Implementation of validated measures both regarding stress (Perceived Stress Questionnaire) and subjective impairments through physical and psychological symptoms (Brief Symptom Inventory).

- Specially tailored training programme in accordance and consultation with student teachers during their teaching practice.

- International generalisation is ambiguous due to different educational systems

- Modularised training concept ensures adaptability to different contexts.

for stress and psychological diseases. In an analysis of 67 occupations, 7 teaching professions showed the highest scores regarding poor mental health. ${ }^{1}$ The proportion of early retirement among teachers is higher than in other academic professions, and fewer teachers reach regular pensionable age. ${ }^{2}$ Mental and psychosomatic illness constitutes the most common reason for early retirement, at about $50 \% .^{23}$ Various studies have shown that a large number of teachers suffer from health problems. For example, Hinz and colleagues found that 29.8\% of 1074 examined teachers in Germany show mental health problems (General Health Questionnaire; GHQ-12 $\geq 4$ ), which is significantly higher than in the German general population $(13.1 \%) .{ }^{4}$ Other studies reported rates of mental health problems among teachers at between $18 \%$ and $29.8 \% .^{5-7}$ Within the population of student teachers, Zimmermann and colleagues even found that $44 \%$ reported impaired mental health. Nevertheless, strained teachers do not appear to be only a German problem, with studies from Italy and Spain reporting depression rates among teachers between $38 \%$ and $49 \%{ }^{89}$ 
The Arbeitsbezogenes Verhaltens- und Erlebensmuster; 'work-related behaviour and experience' (AVEM)) is an instrument that helps to identify four patterns of work-related behaviour and experience measured across 11 dimensions, for example, personal meaning of work, striving for perfection and willingness to overspend. Several studies by Schaarschmidt and colleagues found high percentages for the two risk patterns A (over-engagement) and B (burnout) and low percentages of $\mathrm{G}$ (healthy) within the population of teachers. ${ }^{10-13}$ In 2005, the authors compared a sample of 7693 teachers from Germany with teachers from Austria, Russia, Poland and England, as well as with other professions. They found that the teaching profession showed the most unfavourable pattern distribution (59\% showed risk pattern A or B). This was also the case for teachers from the other evaluated countries. ${ }^{10}$ Even among student teachers, the risk patterns were already highly represented $(40.9 \%){ }^{14}$ Accordingly, the aforementioned studies indicate that a large number of teachers show work-related behaviour that is strongly associated with psychological illness.

Other studies not only investigated the distribution of the AVEM risk patterns, but also assessed how many teachers actually fulfil symptoms of burnout syndrome. ${ }^{15}$ In a study by Körner, fulfilling the criteria for burnout was defined as reaching the clinical cut-off on the Maslach Burnout Inventory (MBI). The authors found that 25.13\% of their sample of grammar school teachers exceeded the clinical cut-off. ${ }^{16}$ The highest proportion of teachers (approximately 24\%) showed conspicuities on the scale 'emotional tiredness', which was also reported in another study. ${ }^{9}{ }^{16}$ Moreover, a further study revealed that student teachers reported even higher rates of 'emotional tiredness' than did qualified teachers. ${ }^{17}$

Nevertheless, being a teacher does not necessarily lead to psychological problems and many teachers seem to cope well with their daily hassles. The question therefore arises of what makes some teachers ill and what keeps some teachers healthy.

Especially among student teachers, external stressors such as 'student characteristics' $(50 \%-70 \%)$, 'classroom practices' $(35 \%-50 \%)$ and 'pressure of examination' (35\%-90\%) are experienced as highly psychologically demanding. ${ }^{18}$ Not only during teacher training, but also in later professional life, teachers are strongly burdened by external stressors. Personal offences, problematic student behaviour and complaints from parents are associated with increased psychological burden. ${ }^{6919}$ All of these studies show, however, that the way in which teachers are influenced by external stressors is strongly associated with their personal resources and coping styles.

For example, Döring-Seipel and Deiber found that teachers with poor or good mental health were distinguished not by objective working conditions or school-related parameters, but rather by the way in which they handled and appraised demands. ${ }^{18}$ Of particular importance in this regard are personal and social resources. Personal resources such as mindfulness, self-efficacy, emotional stability, ability to distance oneself and tolerance of insecurity help teachers to deal with job demands. Moreover, they also positively affect the quality of the students' education. Hinds and colleagues investigated the role of experiential avoidance for stress. Experiential avoidance is the tendency to avoid internal experiences like thoughts and feelings even if doing so causes negative consequences. The authors found that experiential avoidance correlated with depression and burnout symptoms, and that it mediated the relationship between external stressors and personal well-being. ${ }^{9}$ Schmitz et al found that excessive self-demands at the beginning of the teaching career are a risk factor for later invalidity. ${ }^{9}$

In sum, previous studies found that external and internal stressors are responsible for stress-related mental health problems, but there are also some helpful personal resources that can alleviate the influence of these stressors. Since external and some of the internal stressors are not easy to change, it would appear to be beneficial to improve personal skills.

Scientific research on this topic in Germany is somewhat limited. The most well-known projects which examined stress in teachers and developed stress reduction programmes are the 'Freiburger Lehrercoaching' (Unterbrink \& Bauer) and the 'Potsdamer Modell' (Schaarschmidt). ${ }^{20}{ }^{21}$ The 'Freiburger Lehrercoaching' focuses particularly on building professional relationships and communication. By learning to solve interpersonal situations constructively, teachers' stresses and strains are removed and psychological well-being is improved. Studies assessing this programme showed a significant reduction in teachers' scores on the GHQ-12, the Symptom Checklist-90-Revised; SCL90-R (General Severity Index (GSI)) and the MBI compared with a control group. ${ }^{15} 22$ The 'Potsdamer Modell' consists of the components cluster analysis, solution analysis, time and self-management, goal-setting and relaxation. The training has been found to exert positive effects on the AVEM patterns (decreases in patterns $A$ and $B$ and increases in pattern $G$ ), while risk patterns A and B even increased in a control group. Additionally, the intervention group (teachers and student teachers) showed improvement in psychological and medical well-being and affective symptoms such as headaches, lack of concentration, etc. ${ }^{21}$

There are further types of stress reduction training programmes which focus more on cognitive restructuring. For example, Jansen and colleagues showed that a stress resistance training programme addressing the topics of appreciation of stress and cognitive restructuring of stressful thoughts increased the stress sensitivity and competences in stress management. ${ }^{23}$ Another study examined the influence of psychoeducation, cognitive stress management (in line with Kaluza), problem-solving and resource-focusing (Arbeit und Gesundheit im Lehrerberuf, 'work and health in teaching professionals' (AGIL) programme) on depression. ${ }^{24}$ In the study, a group of teachers with psychosomatic symptoms received training using the AGIL programme. At the start of the 
study, 27\% of teachers had high levels of psychological burden (MBI $>14)$ and $73 \%$ had low levels $(\mathrm{MBI} \leq 14)$. In the sample of burdened teachers (indexed prevention), the authors reported a significant decrease in the MBI scores ( $53 \%$ with BMI $\leq 14,12$ month follow-up: $59 \%$ ). Within the sample of unburdened teachers (universal prevention), no significant changes were found.

The aforementioned studies demonstrate that stress prevention training that focuses on cognitive-behavioural or communication strategies is efficient in reducing stress-related symptoms and in increasing personal wellbeing. However, these studies do not cover the construct of mindfulness, even though research has already documented its positive influence. ${ }^{18}{ }^{25}$ Moreover, mindfulness is an effective strategy against experiential avoidance, which is one of the risk factors for depression and burnout symptoms. ${ }^{9}$ Mindfulness is defined as awareness that arises through purposely paying attention, in the present moment, non-judgementally. It is about knowing what is on your mind, ${ }^{26}$ and should be helpful for identifying stressful thoughts and finding helpful solutions. Mindfulness has become a very important tool in stress interventions over the last decade. In particular, Jon Kabat-Zinn's mindfulness-based stress reduction (MBSR) training has made a major contribution to the public awareness of mindfulness. ${ }^{27}$ In a meta-analysis from 2015, Khoury and colleagues evaluated the efficacy of MBSR in healthy individuals. They included 29 studies published in English-language journals and found large effects on stress, and moderate effects on anxiety, depression, distress and quality of life. Only small effects were found on burnout. ${ }^{28}$ Additionally, mindfulness positively affects psychological (measured with the Brief Symptom Inventory (BSI)) and medical complaints, and increases personal well-being, with the frequency of practice at home being especially important. ${ }^{29}$

Other studies revealed that training in mindfulness reduces academic evaluation anxiety, ${ }^{30}$ and some of the aforementioned studies showed that pressure of examination is one of the highest demands for student teachers, thus suggesting that student teachers can also benefit from the positive effects of mindfulness. Shapiro et al found a positive effect of MBSR on the stress of therapists in training, who have to deal with similar demands to student teachers such as particular emotional challenges, interpersonal conflicts and pressure of examination. ${ }^{32}$

So far, mindfulness training interventions for teachers (and student teachers) have only been developed and evaluated in English-speaking countries. For example, Poulin and colleagues developed the Mindfulness-Based Wellness Education (MBWE) programme and demonstrated that MBWE training increased mindfulness and teaching self-efficacy compared with a control group. In turn, this has a positive effect on physical health ratings. ${ }^{33}$ Another training programme including mindfulness in teacher training is the Cultivating Awareness and Resilience in Education (CARE) programme. After participating in CARE, teachers showed significant improvements in teacher well-being, efficacy, time-related stress and mindfulness compared with a control group. Additionally, they were able to better manage their classrooms and build supportive relationships with students. ${ }^{34}$ A previously executed pilot study also illustrated promising results for student teachers. ${ }^{35}$

In a review of the current scientific investigations on this subject conducted in 2017, Kaerner and colleagues found only eight studies (none in Germany) dealing with the topic of MBSR for teachers. All of these studies provided evidence of stress reduction through mindfulness training. ${ }^{36}$ The review showed that further research is severely needed. To date, no such studies are available in Germany.

Research shows that both Cognitive Behavioral Therapy (CBT) strategies and mindfulness have a positive effect on stress and stress-related symptoms. However, there is barely any evidence on the two strategies in combination.

In a recent study, Cook and colleagues emphasised the importance of combining 'multiple theoretical paradigms and corresponding practices'. ${ }^{37}$ In their 'ACHIEVER resilience curriculum' (ARC) training, they combined strategies from CBT, acceptance and commitment therapy (ACT) (especially mindfulness and values clarification) and positive psychology. Using a randomised controlled trial (RCT) design, they found a medium effect of ARC on reducing job-related stress, improving teaching self-efficacy, and on increasing intentions to implement evidence-based classroom practices.

This overview of the current state of research indicates that stress prevention training for teachers in Germany is not yet well established. A combination of CBT and mindfulness-based strategies is not yet available. Research findings on student teachers in Germany are even more sparse. There have been almost no investigations on the psychological burdens, with which student teachers need to deal during their teaching practice. Moreover, to the best of our knowledge, there is currently no special prevention training that addresses their specific requirements.

The aim of the present study is to evaluate a stress prevention training programme which addresses the specific requirements of student teachers by teaching them mindfulness-based and cognitive-behavioural strategies for stress reduction.

\section{METHODS AND ANALYSIS}

The startklar study is designed as a block-RCT with an experimental group and a waiting control group. ${ }^{38}$ Four assessments are scheduled (baseline, post-intervention I, 3-month follow-up, post-intervention II (only control group)), which will allow the evaluation of short-term and medium-term effects of the stress prevention training startklar. For a detailed overview of the trial procedure, see figure 1 . 


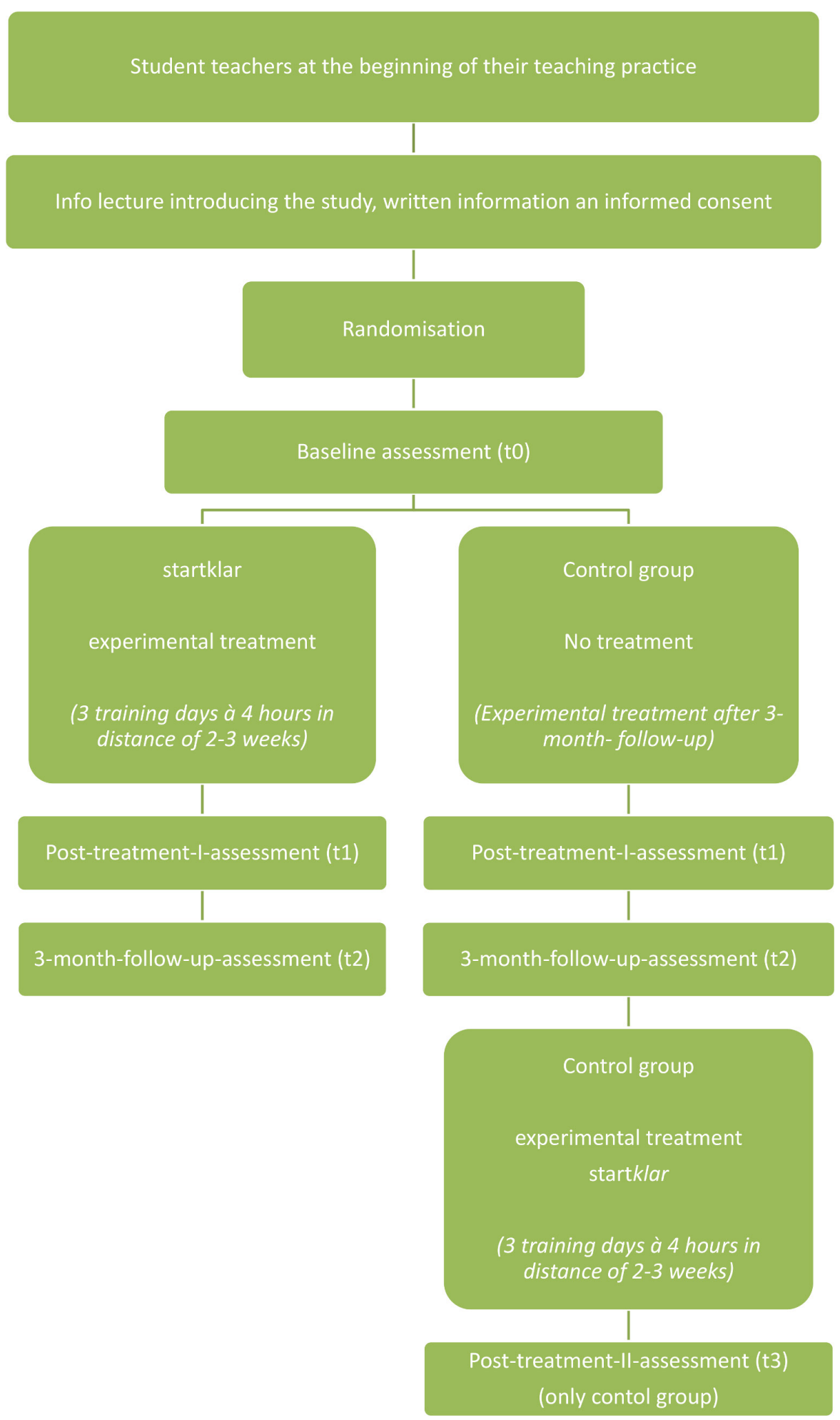

Figure 1 Study schedule.

\section{Participants and recruitment}

Participants are student teachers in their 18-month teaching practice. In Germany, this second practical education period follows university education after the first state examination. The recruitment of the participants will take place in the first threemonths of the teaching practice. In general, the experimental group will begin in the first half of their teaching practice and the control group in the second half. To ensure maximum accuracy and to minimise confounding issues (eg, requirements of the teaching practice, teaching experience), the experimental and control group in every teacher training college will be recruited from one cohort. The recruitment will take place at an information event in the teacher training college. All student teachers from one cohort will be informed about the research project, 
including the detailed procedure of the study and the content of the training. They will also be informed about the monetary incentive they will receive after answering the questionnaires (10 euros for baseline assessment, 20 euros for each of the other assessment points).

In each cohort, we expect to recruit between 80 and 110 student teachers. To achieve the targeted sample size of startklar, which is described further in the sample size calculation, we need to recruit between 20 and 30 participants (10-15 persons per condition) in one teacher training college. Four teacher training colleges will be included for both the experimental and the control group. The experimental and the waiting control group will therefore be composed of four groups each. There are no explicit exclusion criteria for participation. Inclusion criteria are affiliation with one of the participating teacher training colleges and written informed consent.

Subsequently, participants will have the opportunity to ask further questions and can decide whether they wish to participate. Study enrolment will be completed on signing the declaration of consent.

In order to increase the sample size in terms of prevalence estimates, student teachers who do not wish to participate will be invited to fill out the baseline assessment completely anonymously.

\section{Participants and public involvement}

After theory-based development of the training, we consulted student teachers in order to check the content of the training with regard to relevance and feasibility. As a result of this process, we added the additional module 'time management' as this was requested by the participants.

If the participants are interested in receiving personal study results, they will have the possibility to contact study personnel for feedback. Additionally, we will publish the results not only in scientific journals, but also in magazines of our cooperation partner. These magazines are widely distributed in teacher training colleges and schools. After participating in the training, student teachers will be asked to rate their satisfaction with and the feasibility of the training and to state whether they would recommend it to others.

\section{Randomisation}

Before the baseline assessment, the participants will be randomised stratified by gender and allocated either to the experimental or control group. The randomisation will be performed separately for each teacher training college. The randomisation lists will be electronically created by a website (https:// www.randomizer.org) and the participants will be equally distributed to one of the two groups (experimental or waiting control), with a maximum group size of 15 per teacher training college. Allocation cannot be concealed because the control group is a 'passive' waiting control group. For the same reason, it is not feasible to blind the trainers to the group affiliation.
Questionnaires for the participants will be answered via an online platform. In this way, the assessment of the outcomes can be concealed from the trainer and study personnel.

\section{Primary and secondary outcomes}

As already announced in the trial registration, the primary outcome is the mean value of perceived stress (PSQ) ${ }^{39-41}$ A higher PSQ overall score (average score of all items) indicates a higher individual stress experience. It will be measured for all measurement points in order to determine how the experimental group and the control group differ in their perceived stress level. It is expected that after the startklar training the experimental group will report lower mean values than the control group. The secondary outcome is the self-reported subjective psychological symptom burden, measured with the BSI. ${ }^{42-44}$ The BSI provides symptom scores for the nine subscales and an overall score (Global Severity Index (GSI)) assessing psychological distress. Higher scores indicate greater manifestation of symptoms or higher psychological distress. Potentially, the effect can only be measurable at the 3-month follow-up. Additionally, we will capture symptoms of burnout syndrome (emotional depletion, reduced personal performance, depersonalisation) measured by the 'Maslach Burnout Inventory' (MBI-D) ${ }^{45}$ and perceived self-efficacy measured by the 'Allgemeine Selbstwirksamkeit Kurzskala' (ASKU). ${ }^{46} 47$

\section{Trainers}

The training will be performed by two trainers who are skilled in mindfulness meditation, cognitive-behavioural therapy interventions and conducting psychotherapy in groups. They are also qualified in imparting the manualised contents of the startklar training to the participants. Accuracy of the implementation will be secured through intense discussion between the two trainers.

\section{Intervention (startklar- achtsamkeitsbasierte Stresspraevention fuer LehramtsanwaerterInnen)}

The training programme is a modularised intervention encompassing seven modules with different thematic focuses, including mindfulness meditation, acceptance, dysfunctional stress-related cognitive attitudes, automatic thoughts and additional time management strategies. It is based on a previous stress prevention programme for medical students ('MediMind') with fundamental modifications. ${ }^{48} 49$ The content will be provided on three training days (4hours each) over a period of $6-8$ weeks, with 2-3weeks between training days. The first six modules are aligned according to the RAIN principle. ${ }^{50}$ RAIN is a mindfulness tool with four steps which should be helpful in coping with intense and difficult emotions. The acronym stands for first: Recognise what is happening (R); second: Allow life to be just as it is (A); third: Investigate inner experiences with kindness (I); and fourth: Non-identification $(\mathrm{N})$. In the startklar project, we modified this to the 'Weg zur schoenen Aussicht' (Path to the 


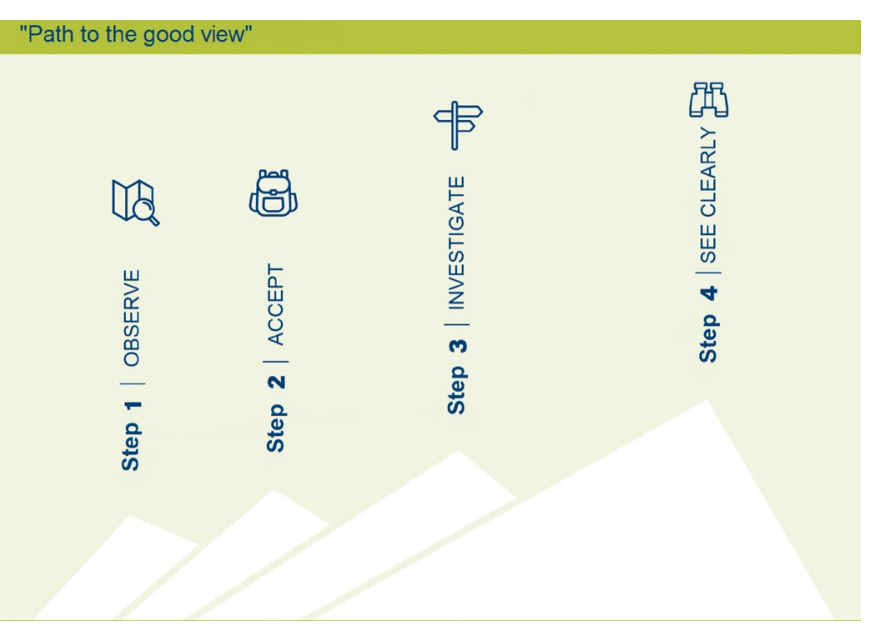

Figure 2 Path to the good view.

good view; figure 2). The aim of this model is to entrench mindfulness into participants' everyday lives in a step-bystep manner.

Every module starts and ends with a mindfulness exercise. Time management strategies form an additional module on the last training day. At the end of each training day, written exercises will be provided and participants will be encouraged to practice mindfulness and deepen the discussed topics. The training is supported by an accompanying booklet received by every participant, which also provides additional information. The participants will be encouraged to frequently practise and complete the administered homework, although no exact amount of practice will be suggested. Participants will report the frequency and further details of practice at post-intervention and 3-month follow-up to control for potential effects.

\section{Module I: introduction}

The first module starts with group interaction practices to enhance group cohesion. ${ }^{51}$ Subsequently, we clarify the necessity of stress prevention training by discussing current research about increased stress experiences in the population of student teachers/teachers and the association with psychological morbidity. ${ }^{15} 1214$ We complete this section by compiling a collection of the participants' personal stressors. 'Mr. Caveman' and 'Riko Roos' are introduced via audio file. These represent two different stress-related cognitive attitudes and automatic thoughts that are involved in the individual emergence of stress. The two characters will appear in different situations across the whole training.

\section{Module II: mindfulness}

The second module introduces the concept of mindfulness with historical information and by outlining its relevance with regard to stress and stress prevention. ${ }^{27} 52$ Mindfulness is then conveyed according to John Kabat$\mathrm{Zinn}^{26}$ with the global principles of mindfulness (awareness, being in the present moment, acceptance) in a first exercise. The student teachers are introduced to the model 'Weg zur schoenen Aussicht' with its different steps (observe, accept, investigate, see clearly), which will be further emphasised in the next modules. With the model, participants should learn to 'bring distance between me and my thoughts', analogous to the defusion concept described in $\mathrm{ACT}^{53}$ as another central principle of mindfulness. ${ }^{54}$

\section{Module III: observe and accept}

In this module, the participants experience the importance of being aware of the present moment. ${ }^{2755}$ Through behavioural experiments and examples from participants' everyday lives, the focus is placed on the negative (and positive) consequences of the 'autopilot mode' and its influence on perceived stress. ${ }^{276}$ As a first strategy to handle negative and unwelcome thoughts, the participants are introduced to the concept of acceptance. ${ }^{53} 5457$ For this, two behavioural experiences ('Don't think about a pink elephant!' and 'Tauziehen mit dem Sorgenmonster' ('tug of war with the worry monster)) are planned, to demonstrate what happens if one is not willing to accept. ${ }^{58}$ The module closes with the exercise of mindful breathing.

\section{Module IV: investigate I}

Module IV is about appreciating the connection between dysfunctional cognitive attitudes, automatic thoughts or judgements, and personal stress experiences. For this purpose, a stress induction exercise is conducted in line with Kaluza et al. ${ }^{59}$ Typical cognitive distortions are presented to the student teachers. By using a given example of a school situation, the participants (split into small groups) write down the typical cognitive distortions. ${ }^{6061}$ After that, two additional strategies to handle dysfunctional thoughts (in addition to acceptance) are introduced. The first strategy is getting distance, and several distancing strategies are discussed. ${ }^{58}$ The second strategy is to challenge the dysfunctional thoughts. For this, the functional cognitions called the 'Gute Gegner' (good opponent) are established. ${ }^{62}{ }^{63}$ Again, divided into small groups, the student teachers use these to build alternative thoughts to the prior automatic thoughts.

\section{Module V: investigate II}

The goal of this module is for the student teachers to learn more about their personal mindsets, attitudes and fundamental assumptions. ${ }^{60}$ By collecting the different expectations the student teachers have to meet every day, it is revealed that their own expectations have the greatest impact on their stress perception. By introducing a short questionnaire in accordance with Kaluza et al, the participants are guided to identify idiosyncratic dominant stress-increasing attitudes such as 'be perfect' or 'don't lose control'. ${ }^{59}$ Additionally, we use a video depicting different scenes in the everyday life of a student teacher in order to illustrate protagonists whose personal attitudes are activated in different situations. Afterwards, there is a discussion of how the attitudes influenced individual 
stress levels in the video. At the end of the module, the student teachers are invited to create a new attitude which they would like to include in their lives in the future.

The goal of modules IV and V is, first, to uncover how the participants evaluate stressful situations, and second, to point out other ways to evaluate stressful situations. Participants will learn that there are indications that even the stress-reducing effect of mindfulness is mediated by the reappraisal of stressful situations. ${ }^{25}$

\section{Module Vl: summary}

In this module, the previously taught content is repeated in small groups to achieve an overview of the different topics and to reinforce the findings of the training days. Each group summarises and presents one topic. Following this, every participant is asked to write down his/her own personal take-home message from the training. This concludes the startklar training. The module finishes with a resource-oriented exercise.

\section{Additional module: time management}

The additional module was included on request of the student teachers. In this module, the advantages of adequate self-management and time management are discussed. The participants learn to use the ALPEN method, which stands for 'Aufgaben planen' (plan tasks), 'Laenge einschaetzen' (estimate duration), 'Pufferzeit reservieren' (reserve buffer time), 'Entscheidungen treffen' (make decisions) and 'Nachkontrolle durchfuehren' (double-check). ${ }^{64}$ As a tool to help with 'making decisions', the Eisenhower Principle is introduced and applied to the planning of their next day. ${ }^{64}$ The Eisenhower principle helps to prioritise duties by urgency and relevance.

\section{Assessments}

\section{Socio-demographic variables}

Socio-demographic variables will be collected. The baseline questionnaire (T0) will include questions about gender, age, nationality, family status, number of children, access to teacher training, highest educational attainment, period since starting teacher training, sparetime work, income, physical and mental diseases, alcohol consumption behaviour, preparation time in teacher training and thoughts about quitting teacher training. The subsequently administered questionnaires (T1, T2, T3) include fewer questions, namely gender, age, assigned cohort, onset of psychotherapy and thoughts about quitting teacher training.

\section{Adherence}

After completion of the training, the following questions will be asked in both groups: number of training lessons attended, number of training strategies used between training lessons, perceived benefit of the training and whether the participants would recommend the training to others (to capture the training satisfaction).

\section{Perceived Stress Questionnaire (PSQ)}

The PSQ measures the individual stress experience as well as the individual stress symptoms on four subscales: worries, tension, joy and demands. ${ }^{41}$ Each of these subscales consists of five items. On a 4-point Likert scale (1=almost never, $2=$ =sometimes, $3=$ =ften, $4=$ =usually), respondents indicate how often the presented statements applied to themselves during the past four weeks. Higher scores on the subscales of worries, tension and demands indicate a higher level of perceived stress, whereas a higher score for joy indicates a lower level of perceived stress. An average score (PSQ Overall Score) of all item scores can be calculated to enable a global assessment of the individual stress experience. After a linear transformation, the overall score as well as the scale values range from 0 to 1 . The English version of the PSQ was translated, revised and evaluated for the German-language area by Fliege $e t a l .{ }^{39} 40$ This shortened German version consists of only 20 items rather than the original 30 items. In exploratory and confirmatory factor analysis, four instead of seven distinct factors can be extracted. Normative data are available for a sample of healthy adults $(n=334)$, undergraduates $(n=249)$, as well as different patient groups, but no gender-specific normative data are available. The internal consistency for the overall score based on the different samples can be rated as satisfactory and ranges from $\alpha=0.85$ to $\alpha=0.94$. For the different subscales, internal consistency across all samples ranges from $\alpha=0.75$ to $\alpha=0.89$, which can be rated as acceptable to good reliability. ${ }^{39}$ The construct validity can also be confirmed due to significant group differences as well as reasonable correlations with different stress-related test procedures. $^{39}$

\section{Brief Symptom Inventory}

The 53-item German version of the BSI is a self-report symptom checklist. ${ }^{44}$ It consists of items selected from the Symptom Checklist 90 Revised (SCL-90-R) to reflect nine subscales assessing the subjective impairments due to physical and psychological symptoms. The nine dimensions measured are somatisation, obsessive-compulsive, interpersonal sensitivity, depression, anxiety, hostility, phobic anxiety, paranoid ideation and psychoticism. On a 5-point Likert scale $(0=$ not at all, $1=$ a little bit, $2=$ moderately, $3=$ quite a bit and $4=$ extremely), respondents indicate their level of distress during the previous seven days. The BSI provides symptom scores for the nine subscales and an overall score (GSI) assessing the psychological distress. The score of each subscale is the average rating of the corresponding items. Higher scores indicate greater manifestation of symptoms. Using gender-specific normative data proposed by Derogatis and adopted in the German version, scores are transformed into T scores to identify cases of distress ( $T \geq 63$ in GSI or on at least two subscales). ${ }^{43}$ The performance and scoring of the German version of the BSI were examined in large samples of participants that included normal, healthy adults $(n=600)$ and undergraduates $(\mathrm{n}=589)$. The internal consistency 
ranges from $\alpha=0.39$ to $\alpha=0.92$ for the sample of healthy adults and from $\alpha=0.59$ to $\alpha=0.95$ for the sample of undergraduates. ${ }^{44}$ For single subscales, good criterion-related validity was demonstrated. The scale structure for the SCL-90-R, which strongly correlated with the BSI $(r=0.92-0.99)$, was confirmed by factor analysis. The scale structure of the BSI has not yet been validated based on a German sample. Expected correlations with other parameters validating the construct validity are available. ${ }^{44}$

\section{Maslach Burnout Inventory}

The German version of the revised MBI is a self-report questionnaire assessing burnout as a multidimensional syndrome. ${ }^{456566}$ It consists of the three subscales emotional exhaustion, depersonalisation and personal accomplishment, and the optional scale involvement (for scaling, cf. Büssing, Perrar, \& Glaser, 2016). Altogether, the MBI encompasses 25 items. $^{22}$ Respondents indicate on a 5-point Likert scale ('very rare' to 'very often') how often the presented statements about emotional and health-related outcomes of strain in everyday work applied to themselves. Furthermore, it is possible to indicate whether an item does not match one's work. Lower scores on the subscales of emotional exhaustion, depersonalisation and involvement correspond to a lower degree of the respective burnout dimension, whereas a lower score on the subscale personal accomplishment corresponds to a higher degree. In line with existing publications, the items are adapted to an everyday school context, substituting patient with student. In terms of evaluation of the MBI scales, separate global scores are provided for each burnout dimension. ${ }^{45}$ The MBI manual presents numerical cut-off points for interpretation. ${ }^{65}$ However, these are mostly arbitrary, and clinically validated cut-off scores remain to be acquired. For the subscale emotional exhaustion, the internal consistency can be rated as good, at $\alpha=0.88$. For the other subscales, internal consistencies cannot be seen as acceptable (personal accomplishment: $\alpha=0.61$, depersonalisation: $\alpha=0.58$ and involvement: $\alpha=0.50)$. Based on correlations with burnout-related constructs, the convergent and discriminant validity of the MBI-D can be rated as acceptable. Despite a four-factor resolution based on a principal component analysis, the factor structure does not match the postulated scale structure. ${ }^{45}$ Due to the optional nature of the involvement subscale, and to shorten the study questionnaire, this subscale was excluded.

\section{Allgemeine Selbstwirksamkeit Kurzskala}

The ASKU is a German-language self-report questionnaire measuring subjectively perceived competences. ${ }^{46} \mathrm{It}$ consists of three items which represent one scale. Respondents indicate on a 5-point Likert scale (1=doesn't apply at all, 2=applies a bit, 3=applies somewhat, 4=applies mostly, 5 =applies completely) how much they agree with the presented statements. The characteristic values for reliability range from $\omega=0.81$ to $\omega=0.86$ (omega-coefficient by McDonald) and can therefore be rated as acceptable. ${ }^{67}$
A confirmatory factor analysis confirmed the one-factor scale structure. Furthermore, strong correlations with other questionnaires on perceived self-efficacy have been found, ${ }^{46}$ indicating good convergent validity.

\section{Sample size calculation}

The sample size was calculated with regard to the primary outcome (perceived stress) with $80 \%$ power and $\alpha=0.05$ significance level. The study by Ebert et al was used as the primary reference as it is the only German study investigating stress reduction training to use PSQ as the primary outcome. ${ }^{68}$ We supplemented the calculation with the study by Pixner, which used the MBI as the primary outcome, and merged effect sizes. ${ }^{22}$ Based on this information, $\mathrm{G}^{*}$ Power V.3.1 was used to determine the required sample size, which resulted in 96 participants.

\section{Statistical analyses}

Descriptive statistics and data quality assessment

At baseline, we will calculate descriptive statistics for both the experimental and the waiting control group and investigate potential differences between the two groups using inferential statistics. ${ }^{69}$ Means, SD, information about data quality and randomness of missing data according to Tabachnik and Fidell will be presented for socio-demographic variables, primary and secondary outcome and additional measures across baseline, post-intervention I, 3-month follow-up and post-intervention II. ${ }^{70}$ If significant differences between potentially confounding variables (sex, age of participants) should be found, these variables will be included as covariates in inferential statistics.

\section{Perceived stress levels and psychological burden}

Prior to the inferential statistics as part of the data analysis, we will analyse perceived stress levels and the psychological burden of the participants at baseline. Socio-demographic variables concerning the self-report of physical and mental diseases, alcohol consumption behaviour and thoughts about quitting the teacher training will be used for sample characterisation. Standardised values (T-values) of the PSQ and the MBI-D will be analysed with regard to perceived stress and the risk of burnout. T-values of the BSI, especially the GSI, will be used as a measure of the psychological burden. Additionally, the ASKU, measuring self-efficacy, will be integrated as a potentially moderating variable on perceived stress and psychological burden.

\section{Inferential statistics}

Following a conservative approach, the analysis will be based on an intention-to-treat design implementing the last-observation-carried-forward method. The $\alpha$-level will be set at $5 \%$ for the rejection of the null hypothesis, resulting in a $p$ value of $\leq 0.05$. The effectiveness of startklar will be analysed with a group $\times$ time interaction principle. ${ }^{69}$ The primary outcome perceived stress (PSQ; higher scores indicate higher perceived stress) will be evaluated with a repeated-measures analysis of variance (ANOVA), in which we expect a significant interaction 
effect at post-intervention I and at 3-month follow-up. ${ }^{40}$ Subjective psychological symptom burden (BSI; higher scores indicate higher psychological symptom burden) as secondary outcome will also be analysed using a repeated-measures ANOVA. ${ }^{44}$ We potentially expect a significant result only at the 3-month follow-up. Additionally, differential changes in burnout syndrome (MBI; higher scores indicate higher emotional depletion, reduced personal performance and higher depersonalisation) and perceived self-efficacy (ASKU; higher scores mean higher perceived self-efficacy) will be evaluated. ${ }^{45}{ }^{46}$ If the assessed data permit, we will additionally consider post-hoc analyses comparing participants with low to moderate burden (universal prevention) and participants with high burden (indicated prevention).

\section{Effect size}

To quantify effect sizes, partial $\eta^{2}$ values, as an indicator in ANOVA designs, will be calculated and cut-off norms will applied according to Cohen's classification. ${ }^{6971}$ Regarding pairwise comparisons, partial $\eta^{2}$ values to denote the interaction effect of group $\times$ time are superior to Cohen's $d$ for the comparison of two variables only. Partial $\eta^{2}$ values will also be used to quantify the influence of potential covariates.

All statistical analyses will be conducted using either SPSS in its latest version (IBM) or Microsoft Office Excel (Microsoft Corporation, Redmond, Washington, USA).

\section{LIMITATIONS}

Several gaps and limitations should be noted in anticipation of the findings of this RCT study. One possible limitation might be a motivational bias of the participants. It might be assumed that only highly motivated student teachers will attend the training. To reduce this potential motivational bias, all participants in the training and the control group will receive a monetary incentive.

We will not measure participants' previous experiences of CBT or mindfulness. Thus, participants who are already highly competent in using CBT or mindfulness strategies may not benefit in the same way. This might lead to an underestimation of the efficacy of the training.

Although participants will be encouraged to practice as frequently as possible and some questions regarding adherence will be included in the post-intervention and at 3-month follow-up, no instructions will be given regarding the frequency and extent of practice between training sessions. Therefore, internal validity may be reduced, although frequency and amount of practice could be introduced as control variables in the analysis.

A further limitation might be the relatively short duration of the intervention, with only 3 days of training within 6 weeks. It might be assumed that steady changes in behaviour and attitudes require more training sessions over a longer period of time. To assess this possibility, a 3-month follow-up is planned to assess the stability of the effects of the training.

\section{IMPLICATIONS}

This block-RCT study aims to evaluate the effects of a mindfulness and cognitive-behavioural-based stress prevention training programme. As part of the data analysis, the perceived stress levels and the psychological burdens of German student teachers will be analysed at baseline.

\section{ETHICS AND DISSEMINATION}

The data handling was approved by the local commissioner for data safety. Elicitation and analysis of study data will take place in a pseudonymised manner. Every participant will receive a study ID which cannot be used to identify the individual. To guarantee confidentiality, the personal data and the questionnaire data will be saved in different places.

The study protocol including the data handling was approved by the ethics committee of the regional medical association in Mainz, Germany (reference number: 837.192.16) and the study is registered in the national study database. All participants will be informed about the study procedure and the aims of the trial. The participants will be informed verbally and in writing at specially arranged information lectures. Participants will be encouraged to ask additional questions. Study participation will be denied if the informed consent form is not signed. Participants can withdraw from the study at any time without further explanation or any negative consequences.

Results will be published through peer-reviewed publications. Additionally, the study protocol and the results will be disseminated through articles in specialist magazines, presentations at specialist conferences for prevention and work safety, and at pertinent congresses.

Acknowledgements The authors are thankful to all participating teacher training colleges and all participants. Florian Kahl and Sarah Mannion de Hernandez proofread the manuscript.

Contributors JE carries out the acquisition of data, operational implementation, and coordination and drafting of the manuscript. SMK developed the intervention program and conceived the study. KK helped with coordination of the trial procedure and drafting the manuscript. FH contributed to the design of the trial and helped in drafting the manuscript. All authors read and approved the final manuscript.

Funding This research was funded by the insurance company, Unfallkasse Rheinland Pfalz, Andernach, Germany .

Competing interests None declared.

Patient consent for publication Not required.

Ethics approval Ethics review board of the local medical association, Mainz, Germany.

Provenance and peer review Not commissioned; externally peer reviewed.

Open access This is an open access article distributed in accordance with the Creative Commons Attribution Non Commercial (CC BY-NC 4.0) license, which permits others to distribute, remix, adapt, build upon this work non-commercially, and license their derivative works on different terms, provided the original work is properly cited, appropriate credit is given, any changes made indicated, and the use is non-commercial. See: http:// creativecommons.org/licenses/by-nc/4.0/. 


\section{REFERENCES}

1. Hasselhorn H-M, Nübling M. Work-related mental fatigue among workers in Germany. Arbeitsmed Sozialmed Umweltmed 2004;39:568-76.

2. Weber A, Weltle D, Lederer P. Early invalidity in the teaching profession: social and occupational medical aspects. German Medical Journal 2004;101:712-8.

3. Weber A, Lederer P. [Morbidity and early retirement among teachers at vocational schools]. Versicherungsmedizin 2006;58:22-8.

4. Hinz A, Zenger M, Brähler E, et al. Effort-reward imbalance and mental health problems in 1074 german teachers, compared with those in the general population. Stress Health 2016;32:224-30.

5. Bauer J, Stamm A, Virnich K, et al. Correlation between burnout syndrome and psychological and psychosomatic symptoms among teachers. Int Arch Occup Environ Health 2006;79:199-204.

6. Bauer J, Unterbrink T, Hack A, et al. Working conditions, adverse events and mental health problems in a sample of 949 German teachers. Int Arch Occup Environ Health 2007;80:442-9.

7. Seibt R, Matz A, Hegewald J, et al. Working conditions of female part-time and full-time teachers in relation to health status. Int Arch Occup Environ Health 2012;85:675-87.

8. Borrelli I, Benevene P, Fiorilli C, et al. Working conditions and mental health in teachers: a preliminary study. Occup Med 2014;64:530-2.

9. Hinds E, Jones LB, Gau JM, et al. Teacher distress and the role of experiential avoidance. Psychol Sch 2015;52:284-97.

10. Schaarschmidt U, ed. Halbtagsjobber? Psychische Gesundheit im Lehrerberuf - Analyse eines veränderungsbedürftigen Zustandes. Weinheim: Beltz, 2005

11. In: Schaarschmidt U, Kieschke U, eds. Gerüstet für den Schulalltag psychologische Unterstützungsangebote für Lehrerinnen und Lehrer. Weinheim/Basel: Beltz, 2007.

12. Schaarschmidt U, Kieschke U, Spörer N, et al. Beanspruchung im Lehrerberuf. In: Hanckel C, Jötten B, Seifried K, eds. Schule zwischen Realität und Vision-Kongressbericht der 14 Bundeskonferenz 2000 in Berlin. Bonn: Deutscher Psychologen Verlag, 2001:135-52.

13. Kieschke U. Arbeit, Persönlichkeit und Gesundheit. Beiträge zu einer differentiellen Psychologie beruflichen Belastungsgeschehens. Berlin: Logos, 2003:378.

14. Zimmermann L, Unterbrink T, Pfeifer R, et al. Mental health and patterns of work-related coping behaviour in a German sample of student teachers: a cross-sectional study. Int Arch Occup Environ Health 2012;85:865-76.

15. Unterbrink T, Hack A, Pfeifer R, et al. Burnout and effort-rewardimbalance in a sample of 949 German teachers. Int Arch Occup Environ Health 2007;80:433-41.

16. Körner SC. Das Phänomen Burnout am Arbeitsplatz Schule- Ein empirischer Beitrag zur Beschreibung des Burnout-Syndroms und seiner Verbreitung sowie zur Analyse von Zusammenhängen und potentiellen Einflußfaktoren auf das Ausbrennen von Gymnasiallehrern [Dissertation]: Universität Erfurt, 2002.

17. Weimar D, Flow-Erleben Sund. Eine empirische Untersuchung zur Bedeutung von Kognitionen, Emotionen und Motivation bei Lehramtsstudierenden, Referendaren und Lehrern. Berlin: Logos, 2005.

18. Döring-Seipel E, Dauber H. Was Lehrerinnen und Lehrer gesund hält: Empirische Ergebnisse zur Bedeutung psychosozialer Ressourcen im Lehrerberuf. Göttingen: Vandenhoeck \& Ruprecht, 2013.

19. Döring-Seipel E, Dauber H. What keeps teachers healthy - The importance of resources, subjective evaluation and handling of the burden on the health of teachers. School Education Today 2010;2.

20. Under Brink T, Bauer J. Teacher health prevention: coaching groups for school teachers according to the Freiburg model. Supervision (42006): System School. Weinheim: Beltz, 2006.

21. Schaarschmidt U, Kieschke U. Potsdam training model with trainees. Teachers and student teachers. In: Schaarschmidt U, Kieschke U, eds. Prepared for everyday school life - psychological support for teachers. Weinheim / Basel: Beltz, 2007.

22. Pixner S. Evaluation of a health prevention measure for BadenWuerttemberg teachers (teacher health project) according to the "Teacher Coaching according to the Freiburg Model" in two presentation modes [Inaugural dissertation]: University of Potsdam, 2014.

23. Jansen LJ. Stress-Resistenz-Training (SRT): Konzeption und Evaluation des Gruppentraingsprogramms zur Verbesserung der Stressresistenz. Berlin: Logos, 2005.

24. Hillert A, Koch S, Kiel E, et al. Mental illness of teachers: occupational therapy and prevention services. Empirical Education 2014;28:190-204.
25. Büttner TR. Mindfulness and health in the teaching profession: relationships between trait mindfulness and stress-related variables. Empirical Education 2014;28:171-89.

26. Kabat-Zinn J. Find peace in everyday life. Frankfurt am Main: Fischer Verlag, 2009.

27. Kabat-Zinn J. Stress management through the practice of mindfulness. Arbor Publisher: Freiburg, 1999.

28. Khoury B, Sharma M, Rush SE, et al. Mindfulness-based stress reduction for healthy individuals: a meta-analysis. J Psychosom Res 2015;78:519-28.

29. Carmody J, Baer RA. Relationships between mindfulness practice and levels of mindfulness, medical and psychological symptoms and well-being in a mindfulness-based stress reduction program. J Behav Med 2008;31:23-33.

30. Dundas I, Thorsheim T, Hjeltnes A, et al. Mindfulness based stress reduction for academic evaluation anxiety: a naturalistic longitudinal study. J College Stud Psychother 2016;30:114-31.

31. Sampl J, Maran T, Furtner MR. A randomized controlled pilot intervention study of a Mindfulness-Based Self-Leadership Training (MBSLT) on stress and performance. Mindfulness 2017;8:1393-407.

32. Shapiro SL, Brown KW, Biegel GM. Teaching self-care to caregivers: Effects of mindfulness-based stress reduction on the mental health of therapists in training. Train Educ Prof Psychol 2007;1:105-15.

33. Poulin PA, Mackenzie CS, Soloway G, et al. Mindfulness training as an evidenced-based approach to reducing stress and promoting well-being among human services professionals. Int $\mathrm{J}$ Health Promot Educ 2008;46:72-80.

34. Jennings PA, Frank JL, Snowberg KE, et al. Improving classroom learning environments by Cultivating Awareness and Resilience in Education (CARE): results of a randomized controlled trial. Sch Psychol Q 2013;28:374-90.

35. Jennings PA, Snowberg K, Coccia MA, et al. Improving classroom learning environments by cultivating awareness and resilience in education (CARE): Results of two pilot studies. Journal of Classroom Interaction 2011;46:37-48.

36. Kaerner T, Müller B, Heinrichs K. Effekte achtsamkeitsbasierter Interventionen zur Verbesserung der Stressbewältigung von Lehrpersonen: eine Zusammenfassung aktueller Forschungsbefunde. Wirtschaft \& \& Erziehung 2017;69:11-16.

37. Cook CR, Miller FG, Fiat A, et al. Promoting secondary teachers' well-being and intentions to implement evidence-based practices: randomized evaluation of the achiever resilience curriculum. Psychol Sch 2017;54:13-28.

38. Grossman J, Mackenzie FJ. The randomized controlled trial: gold standard, or merely standard? Perspect Biol Med 2005;48:516-34.

39. Fliege H, Rose M, Arck P, et al. The Perceived Stress Questionnaire (PSQ) reconsidered: validation and reference values from different clinical and healthy adult samples. Psychosom Med 2005;67:78-88.

40. Fliege $\mathrm{H}$, Rose $\mathrm{M}$, Arck $\mathrm{P}$, et al. Validierung des "Perceived Stress Questionnaire" (PSQ) an einer deutschen Stichprobe. Diagnostica 2001;47:142-52.

41. Levenstein S, Prantera C, Varvo V, et al. Development of the perceived stress questionnaire: a new tool for psychosomatic research. J Psychosom Res 1993;37:19-32.

42. Derogatis LR, Fitzpatrick M. The SCL-90-R, the Brief Symptom Inventory (BSI), and the BSI-18. Maruish ME, ed. The use of psychological testing for treatment planning and outcomes assessment: Instruments for adults. Mahwah, NJ: Lawrence Erlbaum Associates, 2004:1-41.

43. Derogatis LR. BSI brief symptom inventory: administration, scoring, and procedure manual. 4th ed. Minneapolis: MN: National Computer Systems, 1993

44. Franke GH. Brief Symptom Inventory von LR Derogatis (Kurzform der SCL- 90 - R). Deutsche Version. Manual. 1st ed. Göttingen: Beltz Test $\mathrm{GmbH}, 2000$.

45. Buessing A, Perrar KM, Burnout DMvon. Untersuchung einer deutschen Fassung des Maslach Burnout Inventory (MBI-D). Diagnostica 1992;38:328-53.

46. Beierlein C, Kemper CJ, Kovaleva A, et al. Kurzskala zur Erfassung allgemeiner Selbstwirksamkeitserwartungen (ASKU). methoden, daten, analysen 2013;7:251-78.

47. Beierlein C, Kovaleva A, Kemper CJ, et al. Ein Messinstrument zur Erfassung subjektiver Kompetenzerwartungen: Allgemeine Selbstwirksamkeit Kurzskala (ASKU). Mannheim: GESIS - LeibnizInstitut für Sozialwissenschaften, 2012.

48. Kuhlmann SM, Bürger A, Esser G, et al. A mindfulness-based stress prevention training for medical students (MediMind): study protocol for a randomized controlled trial. Trials 2015;16:40.

49. Kuhlmann SM, Huss M, Bürger A, et al. Coping with stress in medical students: results of a randomized controlled trial using a 
mindfulness-based stress prevention training (MediMind) in Germany. BMC Med Educ 2016;16:316.

50. Kornfield J. Bringing home the dharma: awakening right where you are. Boston: Shambala, 2012.

51. Mathieu JE, Kukenberger MR, D'Innocenzo L, et al. Modeling reciprocal team cohesion-performance relationships, as impacted by shared leadership and members' competence. J Appl Psychol 2015;100:713-34.

52. Thichn NH. Das Wunder der Achtsamkeit. 1st edn. Berlin: Theseus, 2006.

53. Hayes SC, Strosahl KD, Wilson KG. Acceptance and commitement therapy: an experimental approach to behavior change. New York: Guilford Press, 1999.

54. Acceptance HSC, Therapy C. Relational frame theory, and the third wave of behavioral and cognitive therapies-republished article. Behavior therapy 2016;47:869-85.

55. Kaltwasser V, Präsenz P. Persönlichkeit und Präsenz. Achtsamkeit im Lehrerberuf. 1st edn. Weinheim \& Basel: Beltz, 2010.

56. Bennett-Levy J, Butler G, Fennell M, et al, eds. Oxford Guide to Behavioural Experiments in Cognitive Therapy. 1st ed. New York: Oxford University Press, 2004

57. Rahl HA, Lindsay EK, Pacilio LE, et al. Brief mindfulness meditation training reduces mind wandering: The critical role of acceptance. Emotion 2017;17:224-30.

58. Wengenroth M. Therapie-Tools Akzeptanz und Commitementtherapie. 2nd edn. Weinheim Basel: Beltz, 2017.

59. Kaluza G. Stressbewätligung. Training manual on psychological health promotion. Berlin Heidelberg: Springer-Verlag, 2011
60. Beck AT, Rush AJ, Shaw BF, et al. Cognitive therapy of depression. New York: The Guilford Press, 1979.

61. Ellis A. Humanistic psychotherapy. The rational-emotive approach. New York: Mcgraw-Hill, 1973.

62. Stavemann HH. Socratic discussion in therapy and counseling: $A$ guide for psychotherapists, counselors and pastoral counselors. 1st edn. Weinheim Basel Berlin: Beltz, 2002.

63. Wilken B. Methods of cognitive restructuring. A Guide to Psychotherapeutic Practice. 7th edn. Stuttgart: Kohlhammer, 2015.

64. Seiwert L. Das $1 \times 1$ des Zeitmanagement. 36th ed. Munich: Graefe and Unzer, 2014.

65. Maslach C, Jackson SE, Leiter MP. Maslach burnout inventory manual. Palo Alto, CA: Consulting Psychologists Press, 1996.

66. Maslach C, Jackson SE. The measurement of experienced burnout. J Organ Behav 1981;2:99-113.

67. McDonald RP. Test theory: a unified treatment. Mahwah: Erlbaum, 1999.

68. Ebert DD, Lehr D, Boß L, et al. Efficacy of an internet-based problem-solving training for teachers: results of a randomized controlled trial. Scand J Work Environ Health 2014;40:582-96.

69. Bortz J. Statistics: for social scientists. 4th ed. Berlin: SpringerVerlag, 2013.

70. Tabachnick B, Fidell L. Using multivariate statistics. Harlow: Pearson Education Limited, 2014.

71. Cohen J. The effect size. Statistical power analysis for the behavioral sciences, 1988:77-83. 